\title{
EXISTENCE THEOREMS CONNECTED WITH THE PICARD- VESSIOT THEORY OF HOMOGENEOUS LINEAR ORDINARY DIFFERENTIAL EQUATIONS
}

\author{
E. R. KOLCHIN
}

1. Introduction. The Picard-Vessiot theory, as recently reformulated by the author, ${ }^{1}$ deals with an abstract ordinary differential field $\mathcal{F}$ of characteristic 0 having an algebraically closed field of constants $\mathcal{C}$, and a differential extension field $G$ over $\mathcal{F}$ with the two properties:

(a) There exists a homogeneous linear differential polynomial $\mathrm{L}(y)=y^{(n)}+p_{1} y^{(n-1)}+\cdots+p_{n} y$ (each $p_{i}$ in 7$)$ which has a fundamental system of solutions $\eta_{1}, \cdots, \eta_{n}$ such that $G=\mathcal{F}\left\langle\eta_{1}, \cdots, \eta_{n}\right\rangle ;^{2}$

(b) The field of constants of $G$ is $\mathcal{C}$.

Such a $G$ is called a Picard-Vessiot extension of $\mathcal{F}$. It is to be noted that the extension $G$ is given, and the existence of the differential polynomial $L(y)$ with the properties (a) and (b) is postulated. It is not immediately apparent, and it would be of interest to know, whether a given $L(y)$, with coefficients $p_{\boldsymbol{i}}$ in $\mathcal{F}$, always has a fundamental system of solutions $\eta_{1}, \cdots, \eta_{n}$ such that $\mathcal{F}\left\langle\eta_{1}, \cdots, \eta_{n}\right\rangle$ is a Picard-Vessiot extension of $\mathcal{F}$ (that is, contains no constant not in $\mathcal{C}$ ). This question was posed by R. Baer (in his critical note on the then current status of the Picard-Vessiot theory, included among comments by $\mathrm{O}$. Haupt in F. Klein's Vorlesungen über hypergeometrische Funktionen, Berlin, 1933), who remarked that the difficulty lay not in proving the existence of a fundamental system of solutions (see PV, §15), but in proving the existence of one which brings in no new constants.

A differential extension field $\mathfrak{F}$ of $\mathcal{F}$ may be an extension of $\mathcal{F}$ by integrals, exponentials of integrals, and algebraic functions. If it is, and if the field of constants of $\mathfrak{T C}$ is still $\mathcal{C}$, then $\mathcal{F}$ is called a liouvillian extension of $\mathcal{F}$. The Picard-Vessiot theory provides a group-theoretic answer to the question of when a Picard-Vessiot extension $G$ of $\mathcal{F}$ is

Presented to the Society, April 17, 1948; received by the editors November 7, 1947.

1 Algebraic matric groups and the Picard-Vessiot theory of homogeneous linear ordinary differential equations, Ann. of Math. (2) vol. 49 (1948) pp. 1-42. This paper, referred to below as "PV", contains the necessary background for the present note.

${ }^{2}$ The notation $\mathcal{F}\langle\ldots\rangle$ indicates, as usual, differential field adjunction. Thus $\mathcal{F}\left\langle\eta_{1}, \cdots, \eta_{n}\right\rangle$ is the differential field consisting of all differential rational functions of $\eta_{1}, \cdots, \eta_{n}$ with coefficients in $\mathcal{F}$. 
(contained in) a liouvillian extension $\mathfrak{H C}(\mathrm{PV}, \S 25)$. It is natural to broaden this question in two respects: first, by relinquishing the requirement that the field of constants of $\pi$ be $\mathcal{C}$ (that is, by demanding only that $\mathfrak{H C}$ be an extension of $\mathcal{F}$ by integrals, exponentials of integrals, and algebraic functions, without demanding that TC be liouvillian); second, by inquiring whether or not $L(y)$ has at least one solution contained in such an extension 3 .

It is the purpose of the present note to show how answers to the points raised in the two preceding paragraphs can be obtained (see Theorems 2 and 4, below) as corollaries to a general theorem on algebraic differential equations due to J. F. Ritt. This theorem can be formulated in the following way ( $\mathcal{F}$ is an ordinary differential field of characteristic $0, y_{1}, \cdots, y_{n}$ are unknowns, and $m$ is a nonnegative integer less than $n$ ).

RItT's Theorem. Let $\mathrm{II}$ be a prime differential ideal in $\mathcal{F}\left\{y_{1}, \cdots, y_{n}\right\},{ }^{3}$ let $J$ be a differential polynomial in $\mathcal{F}\left\{y_{1}, \cdots, y_{n}\right\}$ but not in $\Pi$, let $\Pi_{0}=\Pi \cap \mathcal{F}\left\{y_{1}, \cdots, y_{m}\right\}$. Then there exists a differential polynomial $J_{0}$ in $\mathcal{F}\left\{y_{1}, \cdots, y_{m}\right\}$ but not in $\Pi_{0}$ such that every solution of $\Pi_{0}$ which is not a solution of $J_{0}$ can be completed ${ }^{4}$ into a solution of $\Pi$ which is not a solution of $J$.

In Ritt's proof (Trans. Amer. Math. Soc. vol. 48 (1940) pp. 542552 : see especially pp. 543-545) it is assumed that $\mathcal{F}$ consists of functions of a complex variable meromorphic in a given region, but it is not very difficult to modify his proof to obtain a purely algebraic one, valid for abstract $\mathcal{F}$.

2. The existence theorems. We work with an ordinary differential field $\mathcal{F}$ of characteristic 0 with an algebraically closed field of constants $\mathcal{C}$.

THEOREM 1. Let $\Sigma$ be a proper subset of $\mathcal{F}\left\{y_{1}, \cdots, y_{n}\right\}$, and let $J$ be a differential polynomial in $\mathcal{F}\left\{y_{1}, \cdots, y_{n}\right\}$ but not in the perfect differential ideal $\{\Sigma\}$. Then $\Sigma$ has a solution $\eta_{1}, \cdots, \eta_{n}$ for which $J \neq 0$ and the field of constants of $\mathcal{F}\left\langle\eta_{1}, \cdots, \eta_{n}\right\rangle$ is $\mathcal{C}$.

Proof. Since $J \notin\{\Sigma\}$, we have $J \notin \Pi$, where $\Pi$ is one of the prime components of $\{\Sigma\}$ (in the representation of $\{\Sigma\}$ as an intersection

${ }^{3}$ The notation $\mathcal{F}\{\cdots\}$ indicates, as usual, differential ring adjunction. Thus $\mathcal{F}\left\{y_{1}, \cdots, y_{n}\right\}$ is the differential ring consisting of all differential polynomials in $y_{1}, \cdots, y_{n}$ with coefficients in $\mathcal{F}$.

${ }^{4}$ Following Ritt, we say that a solution $\eta_{1}, \cdots, \eta_{m}$ of $\Pi_{0}$ can be completed into a solution of $\Pi$ provided there exist elements $\eta_{m+1}, \cdots, \eta_{n}$ in some extension of $\mathcal{F}\left\langle\eta_{1}, \cdots, \eta_{m}\right\rangle$ such that $\eta_{1}, \cdots, \eta_{n}$ is a solution of $\Pi$. 
of prime differential ideals none of which contains any other). It follows that $\Pi$ has a solution $\zeta_{1}, \cdots, \zeta_{n}$, which is not a solution of $J$, such that each $\zeta_{i}$ is differentially algebraic over $\mathcal{F}$. (To see this let $y_{1}, \cdots, y_{m}$ be a complete set of arbitrary unknowns for II; by Ritt's theorem any solution of $\Pi_{m}$ which is not a solution of a certain differential polynomial $J_{0} \notin \Pi_{m}$ can be completed to a solution of $\Pi$ which is not a solution of $J$; but $\Pi_{m}=(0)$, so that any set $\zeta_{1}, \cdots, \zeta_{m}$ of differentially algebraic elements not annulling $J_{0}$ can be completed to a solution $\zeta_{1}, \cdots, \zeta_{n}$ of $\Pi$ not annulling $J$; clearly this solution has the desired property.) We suppose that of all solutions $\zeta_{1}, \cdots, \zeta_{n}$ of $\Pi$, not annulling $J$ and having the property that each $\zeta_{i}$ is differentially algebraic over $\mathcal{F}$, ours leads to the smallest value of $p=$ degree of transcendency of $\mathcal{F}\left\langle\zeta_{1}, \cdots, \zeta_{n}\right\rangle$ over $\mathcal{F}$.

Let $\gamma$ be a constant in $\mathcal{F}\left\langle\zeta_{1}, \cdots, \zeta_{n}\right\rangle$, and suppose that $\gamma$ is transcendental over $\mathcal{C}$. Then $(\mathrm{PV}, \S 14$, Theorem 2$) \gamma$ is transcendental over $\mathcal{F}$, too. Introducing a new unknown $w$, let $\Gamma$ be the prime differential ideal in $\mathcal{F}\left\{w, y_{1}, \cdots, y_{n}\right\}$ with generic solution (PV, §11) $\gamma, \zeta_{1}, \cdots, \zeta_{n}$. Then $\Gamma_{0}=\Gamma \cap \mathcal{F}\{w\}$, which has the generic solution $\gamma$, equals $\left[w^{\prime}\right]$. By Ritt's theorem there is a $J_{0} \in \mathcal{F}\{w\}$, with $J_{0} \in\left[w^{\prime}\right]$, such that every solution of $\left[w^{\prime}\right]$ (that is, any constant) not annulling $J_{0}$ can be completed into a solution of $\Gamma$ not annulling $J$. Since there obviously exists a constant $c \in \mathcal{C}$ for which $J_{0} \neq 0, \Gamma$ has a solution $c, \eta_{1}, \cdots, \eta_{n}$ for which $J \neq 0$. By Gourin's theorem (PV, §13) we have: degree of transcendency of $\mathcal{F}\left\langle c, \eta_{1}, \cdots, \eta_{n}\right\rangle$ over $\mathcal{F}$ is less than degree of transcendency of $\mathcal{F}\left\langle\gamma, \zeta_{1}, \cdots, \zeta_{n}\right\rangle$ over $\mathcal{F}$. Therefore $\eta_{1}, \cdots, \eta_{n}$ is a solution of II not annulling $J$ with the property that each $\eta_{i}$ is differentially algebraic over $\mathcal{F}$, and we have: degree of transcendency of $\mathcal{F}\left\langle\eta_{1}, \cdots, \eta_{n}\right\rangle$ over $\mathcal{F}$ is less than $p$. This contradicts the definition of $p$, shows that $\gamma$ is algebraic over $\mathcal{C}$, and proves the theorem.

Theorem 2. If $L(y)=y^{(n)}+p_{1} y^{(n-1)}+\cdots+p_{n} y$, where each $p_{i} \in \mathcal{F}$, then $L(y)$ has a fundamental system of solutions $\eta_{1}, \cdots, \eta_{n}$ such that the field of constants of $\mathcal{F}\left\langle\eta_{1}, \cdots, \eta_{n}\right\rangle$ is $\mathcal{C}$.

Proof. The wronskian determinant $W\left(y_{1}, \cdots, y_{n}\right)$ is not contained in $\left\{L\left(y_{1}\right), \cdots, L\left(y_{n}\right)\right\}$, as $W\left(y_{1}, \cdots, y_{n}\right)$ is of order $n-1$. By Theorem 1, $\left\{L\left(y_{1}\right), \cdots, L\left(y_{n}\right)\right\}$ has a solution $\eta_{1}, \cdots, \eta_{n}$ such that $W\left(\eta_{1}, \cdots, \eta_{n}\right) \neq 0$ and the field of constants of $\mathcal{F}\left\langle\eta_{1}, \cdots, \eta_{n}\right\rangle$ is $\mathcal{C}$. Since $W\left(\eta_{1}, \cdots, \eta_{n}\right) \neq 0$, the elements $\eta_{1}, \cdots, \eta_{n}$ are linearly independent over constants, and constitute a fundamental system of solutions of $L(y)$.

REMarK. As observed by the referee, Theorem 2 extends to sys tems $L_{i}\left(y_{1}, \cdots, y_{n}\right)=0, i=1, \cdots, n$, where $L_{i}\left(y_{1}, \cdots, y_{n}\right)=y_{i}^{\prime}$ 
$-\sum_{j=1}^{n} a_{i j} y_{j}$ (each $a_{i j} \in \mathcal{F}$ ). It is merely necessary to introduce $n^{2}$ unknowns $y_{i j}$ and to note that $D\left(\cdots, y_{i j}, \cdots\right)=\operatorname{det}\left|y_{i j}\right|$ is of order 0 and therefore not contained in $\left\{\cdots, L_{i}\left(y_{j 1}, \cdots, y_{j n}\right)\right.$, ... . The proof then proceeds as above.

We now consider differential fields which are extensions of $\mathcal{f}$ by integrals, exponentials of integrals, and algebraic functions, that is, extensions of the form $\mathcal{F}\left\langle\alpha_{1}, \cdots, \alpha_{m}\right\rangle$ where either $\alpha_{i}^{\prime} \in \mathcal{F}\left\langle\alpha_{1}, \cdots\right.$, $\left.\alpha_{i-1}\right\rangle$ or $\alpha_{i}^{\prime} / \alpha_{i} \in \mathcal{F}\left\langle\alpha_{1}, \cdots, \alpha_{i-1}\right\rangle$ or $\alpha_{i}$ is algebraic over $\mathcal{F}\left\langle\alpha_{1}, \cdots\right.$, $\left.\alpha_{i-1}\right\rangle, i=1, \cdots, m$.

If $\mathfrak{J C}$ is such an extension of $\mathcal{F}$, and if in addition the field of constants of $\mathfrak{F C}$ is again $\mathcal{C}$, then $\mathfrak{F C}$ is said to be a liouvillian extension of F.

In any case, we introduce (corresponding to $\mathrm{PV}, \S 24$ ) ten types of extensions by integrals, exponentials of integrals and algebraic functions, namely, extensions by

(1) integrals, exponentials of integrals, and algebraic functions,

(2) integrals and exponentials of integrals,

(3) exponentials of integrals,

(4) integrals and algebraic functions,

(5) integrals and radicals,

(6) exponentials of integrals,

(7) integrals,

(8) algebraic functions,

(9) radicals,

(10) rational functions.

Theorem 3. Let $\Sigma \subset \mathcal{F}\left\{y_{1}, \cdots, y_{n}\right\}, J \in \mathcal{F}\left\{y_{1}, \cdots, y_{n}\right\}, J \notin\{\Sigma\}$. If $\Sigma$ has a solution $\zeta_{1}, \cdots, \zeta_{n}$ for which $J \neq 0$ such that $\mathcal{f}\left\langle\zeta_{1}, \cdots, \zeta_{n}\right\rangle$ is contained in an extension of $\mathcal{F}$ of one of the types (1)-(10), then $\Sigma$ has a solution $\eta_{1}, \cdots, \eta_{n}$ for which $J \neq 0$ such that $\mathcal{F}\left\langle\eta_{1}, \cdots, \eta_{n}\right\rangle$ is contained in a liouvillian extension of $\mathcal{F}$ of the same type.

PRoof. Suppose $\mathcal{F}\left\langle\zeta_{1}, \cdots, \zeta_{n}\right\rangle \subseteq \mathcal{F}\left\langle\alpha_{1}, \cdots, \alpha_{m}\right\rangle$, where each $\alpha_{i}$ is, appropriately, either an integral of an element of $\mathcal{f}\left\langle\alpha_{1}, \cdots, \alpha_{i-1}\right\rangle$, an exponential of an integral of such an element, or algebraic over $\mathcal{f}\left\langle\alpha_{1}, \cdots, \alpha_{i-1}\right\rangle$. Then there exist differential polynomials $P_{i}\left(u_{1}, \cdots, u_{m}\right), Q\left(u_{1}, \cdots, u_{m}\right) \in \mathcal{F}\left\{u_{1}, \cdots, u_{m}\right\}$, where $u_{1}, \cdots, u_{m}$ are new unknowns, such that $\zeta_{i}=P_{i}\left(\alpha_{1}, \cdots, \alpha_{m}\right) / Q_{i}\left(\alpha_{1}, \cdots, \alpha_{m}\right)$, $i=1, \cdots, m$. There also exist, for $i=1, \cdots, m$, differential polynomials $M_{i}\left(u_{1}, \cdots, u_{i-1}\right), N_{i}\left(u_{1}, \cdots, u_{i-1}\right) \in \mathcal{F}\left\{u_{1}, \cdots, u_{i-1}\right\}$ such that either $\alpha_{i}^{\prime}=M_{i}\left(\alpha_{1}, \cdots, \alpha_{i-1}\right) / N_{i}\left(\alpha_{1}, \cdots, \alpha_{i-1}\right)$, or $\alpha_{i}^{\prime} / \alpha_{i}$ $=M_{i}\left(\alpha_{1}, \cdots, \alpha_{i-1}\right) / N_{i}\left(\alpha_{1}, \cdots, \alpha_{i-1}\right)$, or $\alpha_{i} N_{i}\left(\alpha_{1}, \cdots, \alpha_{i-1}\right)$ is integral and algebraic over $\mathcal{F}\left\{\alpha_{1}, \cdots, \alpha_{i-1}\right\}$. Let $\Lambda$ be the prime dif- 
ferential ideal in $\mathcal{F}\left\{y_{1}, \cdots, y_{n}, u_{1}, \cdots, u_{m}\right\}$ with generic solution $\zeta_{1}, \cdots, \zeta_{n}, \alpha_{1}, \cdots, \alpha_{m}$. By Theorem $1, \Lambda$ has a solution $\eta_{1}, \cdots, \eta_{n}$, $\beta_{1}, \cdots, \beta_{m}$ for which $J\left(\eta_{1}, \cdots, \eta_{n}\right) Q_{1}\left(\beta_{1}, \cdots, \beta_{m}\right) \cdots$ $Q_{n}\left(\beta_{1}, \cdots, \beta_{m}\right) N_{1} \cdots N_{m}\left(\beta_{1}, \cdots, \beta_{m-1}\right) \beta_{1} \cdots \beta_{m} \neq 0$, such that the field of constants of $\mathcal{F}\left\langle\eta_{1}, \cdots, \eta_{n}, \beta_{1}, \cdots, \beta_{m}\right\rangle$ is $\mathcal{C}$. It is now obvious that $\eta_{1}, \cdots, \eta_{n}$ is a solution of $\Sigma$ not annulling $J$, that $\mathcal{F}\left\langle\eta_{1}, \cdots, \eta_{n}\right\rangle \subseteq \mathcal{F}\left\langle\beta_{1}, \cdots, \beta_{m}\right\rangle$, and that $\mathcal{F}\left\langle\beta_{1}, \cdots, \beta_{m}\right\rangle$ is a liouvillian extension of $\mathcal{F}$ of the same type as $\mathcal{F}\left\langle\alpha_{1}, \cdots, \alpha_{m}\right\rangle$.

A homogeneous linear differential polynomial $L(y)=y^{(n)}+p_{1} y^{(n-1)}$ $+\cdots+p_{n} y$, with each $p_{i}$ in $\mathcal{F}$, is linearly reducible over $\mathcal{F}$ if there exist two homogeneous linear differential polynomials $M(y)$ and $N(y)$ with coefficients in $\mathcal{F}$ and of positive order, such that $L(y)=M(N(y))$. If $L(y)$ is not linearly reducible over $\mathcal{F}$ it is linearly irreducible over $\mathcal{F}$.

THEOREM 4. Let $L(y)=y^{(n)}+p_{1} y^{(n-1)}+\cdots+p_{n} y$, with each $p_{i}$ in $\mathcal{F}$, be linearly irreducible over $\mathcal{F}$. If $L(y)$ has one solution contained in an extension of $\mathcal{F}$ of one of the types (1)-(10), then $L(y)$ has a fundamental system of solutions $\eta_{1}, \cdots, \eta_{n}$ such that $\mathcal{F}\left\langle\eta_{1}, \cdots, \eta_{n}\right\rangle$ is a liouvillian extension of $\mathcal{F}$ of the same type.

Proof. By Theorem 3, $L(y)$ has a solution $\eta$ contained in a liouvillian extension $\mathfrak{F C}$ of $\mathcal{F}$ of the required type. By Theorem 2, $L(y)$ has a fundamental system of solutions $\zeta_{1}, \cdots, \zeta_{n}$ such that the field of constants of $\mathcal{H}\left\langle\zeta_{1}, \cdots, \zeta_{n}\right\rangle$ is $\mathcal{C}$. Therefore $\eta$ is a linear combination over $\mathcal{C}$ of $\zeta_{1}, \cdots, \zeta_{n}$, so that $\mathcal{G}=\mathcal{F}\left\langle\zeta_{1}, \cdots, \zeta_{n}\right\rangle$ is a Picard-Vessiot extension of $\mathcal{F}$ containing $\eta$. Letting (\$) be the group of all automorphisms of $G$ over $\mathcal{F}$, we see that, in the linear space over $\mathcal{C}$ spanned by $\zeta_{1}, \cdots, \zeta_{n}$, the linear subspace spanned by the set of all elements $\sigma \eta(\sigma \in(S)$ is invariant under (S). Since $L(y)$ is linearly irreducible, the only invariant linear subspaces are the zero space and the whole space (PV, §22, Theorem 1). Therefore there exist $n$ automorphisms $\sigma_{1}, \cdots, \sigma_{n}$ in \& such that $\sigma_{1} \eta, \cdots, \sigma_{n} \eta$ are linearly independent over $\mathcal{C}$.

Now, $\sigma_{1}$ can be extended to an isomorphism $\tau_{1}$ of $\mathfrak{H C}_{1}$ $=\mathfrak{H C}\left\langle\zeta_{1}, \cdots, \zeta_{n}\right\rangle$ over $\mathcal{F} ; \sigma_{2}$ can be extended to an isomorphism $\tau_{2}$ of $\mathfrak{F C}_{2}=\mathcal{F}\left\langle\mathcal{F C}_{1}, \tau_{1} \mathfrak{H C}_{1}\right\rangle$ over $\mathcal{F}_{;} \cdots ; \sigma_{n}$ can be extended to an isomorphism $\tau_{n}$ of $\mathfrak{F C}_{n}=\mathcal{F}\left\langle\mathcal{H C}_{n-1}, \tau_{n-1} \mathfrak{H C}_{n-1}\right\rangle$ over $\mathcal{F}$. Therefore $\sigma_{1} \eta, \cdots$, $\sigma_{n} \eta$ is a fundamental system of solutions of $L(y)$ contained in $\mathfrak{F}_{0}=\mathfrak{F}\left\langle\tau_{1} \mathfrak{H C}, \cdots, \tau_{n} \mathfrak{F C}\right\rangle$. That is, $\sigma_{1} \eta, \cdots, \sigma_{n} \eta$ is a solution of $\left\{L\left(y_{1}\right), \cdots, L\left(y_{n}\right)\right\}$ for which $W\left(\sigma_{1} \eta, \cdots, \sigma_{n} \eta\right) \neq 0$, such that $\mathcal{F}\left\langle\sigma_{1} \eta, \cdots, \sigma_{n} \eta\right\rangle \subseteq \mathcal{H}_{0}$. Now, $\mathcal{F}_{0}$ is clearly an extension of $\mathcal{F}$ of the same type that $\mathcal{F C}$ is. Therefore, by Theorem $3,\left\{L\left(y_{1}\right), \cdots, L\left(y_{n}\right)\right\}$ has a solution $\eta_{1}, \cdots, \eta_{n}$ for which $W\left(\eta_{1}, \cdots, \eta_{n}\right) \neq 0$, such that 
$\mathcal{F}\left\langle\eta_{1}, \cdots, \eta_{n}\right\rangle$ is contained in a liouvillian extension of $\mathcal{F}$ of the required type. It follows that $\eta_{1}, \cdots, \eta_{n}$ is a fundamental system of solutions of $L(y)$, and (see PV, §25) $\mathcal{F}\left\langle\eta_{1}, \cdots, \eta_{n}\right\rangle$ is itself a liouvillian extension of $\mathcal{F}$ of the required type.

Columbia University

\section{ON SOME EXAMPLES IN THE THEORY OF POWER SERIES}

\section{P. TURÁN}

Fabry, ${ }^{1}$ Hardy, ${ }^{2}$ S. Bernstein ${ }^{3}$ and Carleman ${ }^{4}$ discovered that for any $\delta>0$ there exist power series $\sum_{0}^{\infty} a_{v} z^{\nu}$ which are continuous for $|z| \leqq 1$ and for which the series $\sum\left|a_{\nu}\right|^{2-\delta}$ diverges. An elegant example is provided by the series

$$
f(z)=\sum_{n=2}^{\infty} \frac{e^{i c n \log n}}{n^{1 / 2} \log ^{\beta} n} z^{n}, \quad c \neq 0, \beta>1,
$$

which is continuous for $|z| \leqq 1$ (and even uniformly convergent there) $;^{5}$ another example for the Carleman singularity, explicitly

Received by the editors July 22, 1947, and, in revised form, November 17, 1947.

${ }^{1}$ E. Fabry, Ordre des points singuliers de la série de Taylor, Acta Math. vol. 36 (1913) pp. 69-194, esp. p. 103.

${ }^{2}$ G. H. Hardy, A theorem concerning Tayor's series, Quarterly Journal of Pure and Applied Mathematics vol. 44 (1913) pp. 147-160. In these two papers it was shown that if $\delta>0$ the series $f_{2}(z)=\sum_{1}^{\infty} e^{i \nu^{1-\delta} / z^{\nu} / \nu^{1-\delta}}$ is uniformly convergent for $|z|<1$ and in Hardy's paper remark was made (p. 157) upon H. Bohr's problem on constructing a power series which is uniformly but not absolutely convergent for $|z| \leqq 1$.

${ }^{3}$ S. Bernstein, C. R. Acad. Sci. Paris (1914). He gave interesting cosine-polynomials $H(x)=\sum_{\nu=1}^{p-1} b_{\nu} \cos \nu x(p$ prime $\equiv 1 \bmod 4)$ with the properties $|H(x)| \leqq 1$ and $\sum_{\nu=1}^{p=1}\left|b_{\nu}\right|=(p-1) / p^{1 / 2}$ which contains the seeds of the Carleman-singularity and were indeed the basis of Carleman's own construction.

${ }^{4} \mathrm{~T}$. Carleman, Über Fourier Koefficienten einer steigen Funktionen, Acta. Math. vol. 41 (1918) pp. 337-384. Here is asserted explicitly and proved for the first time the existence of a continuous $f_{3}(x) \sim \sum\left(a_{\nu} \cos \nu x+b_{\nu} \sin \nu x\right)$ with $\sum_{1}^{\infty}\left(\left|a_{\nu}\right|^{2-\delta}+\left|b_{\nu}\right|^{2-\delta}\right)$ $=\infty, \delta$ arbitrarily small: the existence of a continuous power series with the same property seems to be explicitly mentioned at the first time by Sidon; see footnote 9.

${ }^{5}$ See G. H. Hardy and J. E. Littlewood (Some problems of Diophantine approximation: A remarkable trigonometrical series, Proc. Nat. Acad. Sci. U.S.A. vol. 2 (1916) pp. $583-586)$, who considered only the functions $f_{1}(z)=\sum_{n-2}^{\infty}\left(e^{i c n} \log n / n^{1 / 2+\alpha}\right) z^{n}, c \neq 0$, $0<\alpha<1$ (the divergence of the $(2-\delta)$ th power of the moduli of the coefficients is not explicitly mentioned there). Series (1) seems to have been discussed for the first time by Zygmund in his book on trigonometric series. He uses there (in a simplified form) an argument due to Hille (Note on a power series considered by Hardy and Littlewood, J. London Math. Soc. vol. 4 (1929) pp. 176-182) and based on the application of Van der Corput's estimates to sums of the form $\sum e^{2 \pi i g(n)}$. 